\title{
Predicción computacional de estructura terciaria de las proteínas humanas Hsp27, $\alpha B$-cristalina y HspB8
}

\author{
Homero Sáenz-Suárez ${ }^{1 *}$, Leonardo René Lareo ${ }^{2 \dagger}$, Carlos Oribio-Quinto ${ }^{1}$, \\ Juan Martínez-Mendoza ${ }^{1}$, Aura Chávez-Zobel ${ }^{1}$ \\ ${ }^{1}$ Unidad de Biología Celular y Microscopía. Decanato de Ciencias de la Salud. \\ Universidad Centroccidental Lisandro Alvarado. Barquisimeto, Venezuela. \\ ${ }^{2}$ Grupo de Bioquímica Computacional y Bioinformática, Departamento de Bioquímica, \\ Facultad de Ciencias. Pontificia Universidad Javeriana. Bogotá, D.C. Colombia. \\ *hsaenz@ucla.edu.ve
}

Recibido: 03-11-2010; Aceptado: 04-03-2011

\begin{abstract}
Resumen
Objetivo. Realizar predicciones computacionales de estructura de las proteínas humanas Hsp27, $\alpha \mathrm{B}$ cristalina y HspB8. Materiales y métodos. La predicción de la estructura secundaria se obtuvo mediante un consenso de los programas de predicción secundaria GOR 4, nnPred, Sspro, APSSP2, JPredict, Porter, Prof, SOPMA, HNN y Psi-Pred. Los modelos de estructura terciaria se elaboraron a partir de fragmentos homólogos de proteínas con estructura terciaria conocida que fueron obtenidos por múltiples alineamientos. Empleando la secuencia primaria se obtuvieron perfiles de antigenicidad de las proteínas nativas y fueron analizados los perfiles de hidrofobicidad, polaridad, flexibilidad, accesibilidad tanto de las proteínas nativas como de las mutadas. Resultados. Las predicciones de estructura secundaria y terciaria de las proteínas estudiadas muestran que en los tres casos, más del 65\% son regiones en coil, 20-25\% en hoja plegada y menos del 10\% en alfa hélice. Los análisis de estructura primaria muestran que al menos uno de los perfiles estudiados, en cada mutación está alterado. Conclusiones. Los análisis comparativos de estructura sugieren que las mutaciones afectan la solubilidad de las proteínas mutadas y con ello su función como chaperonas moleculares.
\end{abstract}

Palabras clave: Hsp27, $\alpha$ B-cristalina, HspB8, predicción de estructura secundaria, modelo computacional estructura terciaria.

\begin{abstract}
Computational prediction of tertiary structure of the human proteins Hsp27, $\alpha$ B-crystalline and HspB8. Objective: To make computational predictions of the structure of the human proteins Hsp27, $\alpha$ B-crystalline and HspB8. Materials and methods. The prediction of the secondary structure was obtained by a consensus of the programs for secondary prediction GOR 4, nnPred, Sspro, APSSP2, JPredict, Porter, Prof, SOPMA, HNN and Psi-Pred. The models of tertiary structure were built from fragments homologous to proteins with tertiary known structure that were obtained by multiple alignments. Using the primary sequence we obtained the antigenicity profiles of native proteins and we analyzed the profiles of hydrophobicity, polarity, flexibility and accessibility of both native and mutant proteins. Results. Predictions of the secondary and tertiary structures of the studied proteins show that in the three cases, more than $65 \%$ are coil regions, $20-25 \%$ are folded sheet and less than $10 \%$ are alpha helix. Analyses of the primary structure show that at least one of the studied profiles in every mutation is altered. Conclusions. The comparative analyses of structure suggest that mutations affect the solubility of the mutated proteins and hence affect their function as molecular chaperones
\end{abstract}

Key words: Hsp27, $\alpha$ B-cristalline, HspB8, prediction of secondary structure, computational model of tertiary structure 


\section{Resumo}

Predição computacional da estrutura terciária das proteínas humanas Hsp27, $\alpha$ B-cristalina e HspB8. Objetivo. Realizar predições computacionais da estrutura das proteínas humanas Hsp27, $\alpha \mathrm{B}$-cristalina e HspB8. Materiais e métodos. A predição da estrutura secundária foi obtida através de um consenso dos programas de predição secundária GOR 4, nnPred, Sspro, APSSP2, JPredict, Porter, Prof, SOPMA, HNN e Psi-Pred. Os modelos de estrutura terciária foram desenvolvidos a partir de fragmentos homólogos de proteínas com estrutura terciária conhecida que foram obtidos por alinhamentos múltiplos. Utilizando a sequiência primária foram obtidos perfis de antigenicidade das proteínas nativas e foram analisados os perfis de hidrofobicidade, polaridade, flexibilidade e acessibilidade, tanto da proteína nativa, como das mutantes. Resultados. As predições de estrutura secundária e terciária das proteínas estudadas mostram que nos três casos, mais de $65 \%$ são regiões em coil, 20-25\% de folha pregueada e inferior a 10\% em alfa-hélice. A análise da estrutura primária mostra que pelo menos um dos perfis estudados, em cada mutação está alterado. Conclusões. A análise comparativa da estrutura sugere que as mutações afetam a solubilidade das proteínas mutantes e, assim, sua função como chaperones moleculares.

Palavras-chave: Hsp27, $\alpha$ B-crystalina, HspB8, predição da estrutura secundária, modelo computacional estrutura terciária.

\section{Introducción}

Las proteínas de stress fueron estudiadas por primera vez en 1962 por Ritossa, quien trabajando con células de las glándulas salivares de Drosophila, observó que al utilizar como estímulos el calor, el dinitrofenol y el salicilato de sodio se producía un incremento en la síntesis de proteínas con peso molecular de 70 y $26 \mathrm{kDa}$. Las mismas fueron llamadas heat shock proteins (Hsp) y en 1973 son descritas como proteínas inducidas por el stress (1). Estas proteínas están presentes en todas las células e intervienen durante la síntesis proteica, uniéndose a peptidos nacientes para dirigir su plegamiento, lo que garantiza su estructura tridimensional y consecuentemente su funcionamiento correcto. De igual manera, son capaces de unirse a péptidos y proteínas alteradas por diversos tipos de agresiones, facilitando su reorganización o degradación. Un amplio espectro de agresiones fisiológicas y ambientales como infecciones virales, procesos inflamatorios, cuadros febriles, exposición de las células a citotoxinas, anoxia, choque térmico y $\mathrm{pH}$ ácido, inducen un rápido aumento en las concentraciones de las Hsp. La función como moléculas chaperonas constituye un mecanismo de defensa que permite a la célula adaptarse a condiciones anómalas y aumentar su capacidad de supervivencia (2).

La masa molecular, su comportamiento celular y las funciones que realizan, definen las diferentes familias de Hsp, entre las cuales se encuentran las Hsp100/HSPH, Hsp90/HSPC1, Hsp70/HSPA, Hsp60/HSPD1, Hsp40/ DNAJB1 y Hsp/HSPB (small heat shock protein) $(3,4)$. Las Hsp constituyen una familia formada por 10 proteínas: Hsp27, $\alpha \mathrm{B}$-cristalina, $\alpha \mathrm{A}$-cristalina, Hsp20, MKBP/HspB2, HspL27/HspB3, cvHsp, HspB8, HspB9 y HspB10 (4-11). Para las Hsp, se ha propuesto un mecanismo de chaperonaje molecular que sugiere que esas proteínas intervienen en la protección de polipéptidos mal plegados con la finalidad de bloquear interacciones no apropiadas. Sin embargo, las Hsp no participan en el replegaje de las proteínas desnaturalizadas. De esa manera, la actividad de chaperonas moleculares de esas proteínas difiere de Hsp60 y Hsp70 (12). De otro lado, en función del consumo de energía, se consideran dos grupos, las dependientes de ATP, como Hsp100/HSPH, Hsp90/HSPC1, Hsp70/HSPA, Hsp60/ HSPD1, Hsp40/DNAJB1 y las independientes de ATP como Hsp/HSPB (13).

Los resultados obtenidos en numerosas investigaciones, durante los años 90, sugirieron que las proteínas de estrés se encuentran implicadas no sólo en procesos fisiológicamente normales, sino asociadas a procesos patológicos (15). Las enfermedades conformacionales o proteinopatías, se caracterizan por la presencia citoplasmática de cuerpos de inclusión o agregados proteicos, los cuales, de manera general, son el resultado de proteínas mal plegadas $(11,14)$. En esos cuerpos de inclusión ha sido descrita la presencia de ubiquitina, subunidades del proteosoma y chaperonas moleculares, elementos que participan en los procesos celulares de control de calidad a nivel de las proteínas $(15,16)$. La capacidad de las Hsp para proteger contra la desnaturalización de las proteínas inducida por el estrés térmico y la asociación de las Hsp con los agregados protéicos, sugieren que las chaperonas moleculares están implicadas en el desarrollo de enfermedades neurodegenerativas como Parkinson, Huntington y Alexander (15). Esto último, en la búsqueda de medidas terapéuticas para estas patologías, constituye una potencial alternativa basada en la producción de este tipo de proteínas. Con el fin de generar conocimientos que contribuyan al logro de este propósito, en el presente trabajo mediante el empleo de herramientas computacionales, se analiza la estructura primaria, se predice la estructura secundaria, son elaborados modelos de estructura terciaria de las proteínas Hsp27, alfa B-cristalina y HspB8 humanas y se estudia el efecto de las mutaciones reportadas (17) sobre la estructura de dichas moléculas. Específicamente G84R, L99M, P39L, P182L, R127W, 
R136W, R140G, S135F, T151I sobre la primera, R120G y D140N sobre la segunda y K141N y K141E sobre la tercera de las proteínas.

\section{Materiales y métodos}

En GenBank el número de acceso para Hsp27 es BAB17232.1, para $\alpha \mathrm{B}$-cristalina ACA05949.1 y para HspB8 CAG384884.1, donde se encuentran las secuencias de aminoácidos utilizadas en este trabajo.

\section{Análisis de la estructura primaria}

Para la determinación de los perfiles de accesibilidad, hidrofobicidad, polaridad y flexibilidad se empleó "ProScale" de ExPASy (18), mientras que para la predicción del perfil de antigenicidad se utilizó el programa JaMBW Chapter 3.1.7 (19) y los alineamientos de secuencias fueron realizados empleando el programa HHpred (20). En todos los casos se utilizaron los programas con los parámetros pre-establecidos, sin variación en ningún caso.

\section{Predicción de la estructura secundaria}

En cuanto a la predicción de la estructura secundaria se empleó "Proteomics and Sequence Análisis Tools" de ExPASy (18), para lo cual las secuencias de aminoácidos de Hsp27, $\alpha \mathrm{B}$-cristalina y HspB8 fueron sometidas a los siguientes programas de predicción de estructura secundaria: GOR 4, nnPred, Sspro, APSSP2, JPredict, Porter, Prof, SOPMA, HNN y Psi-Pred (21-28), con el propósito de obtener un consenso de las predicciones de dichos programas.

\section{Predicción de la estructura terciaria}

Para la predicción de la estructura terciaria se empleó el programa HHpred, el cual mediante alineamientos múltiples con proteínas de las cuales existe estructura terciaria obtenida experimentalmente, permitió obtener fragmentos de otras moléculas, con secuencias similares a cada una de las moléculas estudiadas. Estos fragmentos homólogos fueron unidos, empleando el programa Swiss-pdb Viewer versión 4.0.1 (spdbv 4.0.1) (28), y finalmente los residuos sin predicción computacional fueron adicionados manualmente uno a uno, atendiendo a su orientación de acuerdo a la predicción secundaria. En cada caso el modelo de estructura obtenido fue minimizado con el programa Discover 3, del paquete de Insight II 2004, en un computador Silicon Graphic Octane. Para visualizar la estructura tridimensional, se emplearon los programas POV 3.6, RasMol 2.6 (29) y Spdbv 4.0.1 (28).

\section{Análisis de las mutaciones}

Para cada una de las mutaciones fue realizado el mismo análisis de estructura primaria que fue realizado a las proteínas salvajes.

\section{Resultados}

\section{Análisis de la estructura primaria}

La figura 1 muestra las secuencias primarias de las proteínas humanas Hsp27, $\alpha$ B-cristalina y HspB8, las cuales se encuentran en GenBank y fueron obtenidas mediante los números de acceso BAB17232.1, ACA05949.1 y CAG384884.1, respectivamente.

La obtención de los perfiles de hidrofobicidad fue realizada mediante el algoritmo de Kyte-Doolitle, el cual emplea una escala que otorga valores de hidrofobicidad para cada uno de los aminoácidos, con variaciones entre -3.5 (Glutamato) y 4.2 (valina), mientras que para la obtención de los perfiles de polaridad se empleó el algoritmo de Zimmerman, que utiliza una escala que asigna valores de polaridad para cada uno de los aminoácidos, con variaciones entre 0.0 (Alanina) y 52 (Arginina). En el caso del porcentaje de accesibilidad fue utilizada una escala que asigna porcentajes de accesibilidad a cada uno de los aminoácidos, con variaciones entre 0.3 (Lisina) y 0.9 (Cisteina) y finalmente para la flexibilidad fue utilizada la escala de Bhaskaran, la cual otorga valores de flexibilidad para cada uno de los aminoácidos, con variaciones entre 0.300 y 0.540 (18). En todos los casos los algoritmos consideraron ventanas de 9 aminoácidos. Los perfiles obtenidos para Hsp27, alfa B-cristalina y HspB8 muestran valores bajos de hidrofobicidad y altos de polaridad (Figura 2), lo que sugiere que son moléculas poco hidrofóbicas y predominantemente polares. Los altos valores de flexibilidad y accesibilidad, sugieren que son moléculas flexibles y con regiones de alta accesibilidad (Figura 3).

\section{Predicción de la estructura secundaria}

El consenso de los 10 programas computacionales de predicción de estructura secundaria sugieren que Hsp27 tiene una estructura secundaria compuesta por $9.27 \%$ en hélice, $24.39 \%$ en hoja plegada y $66.34 \%$ en coil. En el caso de la alfa B-cristalina $6.86 \%$ en alfa hélice, $27.43 \%$ 
en hoja plegada y $65.71 \%$ en coil, mientras que HspB8 presenta $2.55 \%$ en hélice, $21.43 \%$ en hoja plegada y $76.02 \%$ en coil (Figura 4).

\section{Predicción de la estructura terciaria}

Hsp27: Se efectuaron alineamientos múltiples con proteínas que poseen estructura terciaria determinada experimentalmente y se encontraron 63 fragmentos con identidades desde 7 hasta $38 \%$ y probabilidades desde 20,3 a 99,98\%. Se utilizaron fragmentos de las proteínas 1gme_A, 1hsh_A y 2Bol_A que específicamente corresponden a los aminoácidos en posiciones $12-32 ; 33$ 44 y 45-204 respectivamente y constituyen el 93,4\% del modelo. Empleando el programa Spdbv 4.0.1, estos fragmentos homólogos fueron unidos y finalmente, fueron adicionados manualmente (de acuerdo a la predicción de su estructura secundaria) los 11 aminoácidos iniciales que carecían de predicción de estructura terciaria, es decir, lo correspondiente al 6,6\% restante del modelo (Figura 5A). $\alpha \mathbf{B}$-cristalina: Se efectuaron alineamientos múltiples con proteínas que poseen estructura terciaria determinada experimentalmente y se encontraron 68 fragmentos con identidades desde 6 hasta $33 \%$ y probabilidades desde 20,11 a $99,92 \%$. Se utilizaron fragmentos de las proteínas 1hsh_A, 1gme_A, y 2Bol_A que específicamente corresponden a los aminoácidos en posiciones 9-32; 33165 y 166-175 respectivamente y constituyen el $95,5 \%$ del modelo. Empleando el programa Spdbv 4.0.1, estos fragmentos homólogos fueron unidos y finalmente, fueron adicionados manualmente (de acuerdo a la predicción de su estructura secundaria) los 8 aminoácidos iniciales que carecían de predicción de estructura terciaria, es decir, lo correspondiente al 4,5\% restante del modelo (Figura 5B, 5C).
HspB8: Se efectuaron alineamientos múltiples con proteínas que poseen estructura terciaria determinada experimentalmente y se encontraron 44 fragmentos con identidades desde 10 hasta $33 \%$ y probabilidades desde 20,3 a $99,86 \%$. Se utilizaron fragmentos de las proteínas 1hsh_A, 1gme_A, y 2Bol_A que específicamente corresponden a los aminoácidos en posiciones 14-30; 31 97 y 98-196 respectivamente y constituyen el 93,4\% del modelo. Empleando el programa Spdbv 4.0.1, estos fragmentos homólogos fueron unidos y finalmente, fueron adicionados manualmente (de acuerdo a la predicción de su estructura secundaria) los 13 aminoácidos iniciales que carecían de predicción de estructura terciaria, es decir, lo correspondiente al 6,6\% restante del modelo (Figura 5D).

El extremo N-terminal de Hsp27 que posee el motivo WD/ EPF es la región esencial para la formación de oligómeros. Se estima que la forma oligomérica de Hsp27 probablemente está conformada de dímeros estables, que se forman por interacciones entre los dominios alfacristalinos de monómeros contiguos. Estos dímeros se asocian para formar tetrámeros, los cuales finalmente forman oligómeros inestables. En su forma oligomérica Hsp27 (aproximadamente $800 \mathrm{kDa}$ ), está compuesta por 16 a 32 subunidades y es solamente en esta conformación que posee actividad de chaperona molecular $(30,31,32)$. En el caso de alfa B-cristalina, se estableció que sedimenta en gradiente de glicerol como un oligómero de aproximadamente 500 a $540 \mathrm{kDa}$, dado que el monómero es de $21 \mathrm{kDa}$, la forma oligomérica equivaldría a 24 unidades (33). Igualmente, se conoce que HspB8 sedimenta con un peso molecular de aproximadamente $62 \mathrm{kDa}$, si el monómero es $28 \mathrm{kDa}$, equivaldría a un dímero (30). Las figuras $5 \mathrm{~F}$ y $5 \mathrm{G}$ muestran modelos tetraméricos de Hsp27 y alfa B-cristalina, mientras que la figura $5 \mathrm{H}$ muestra la $\mathrm{HspB} 8$ en su forma dimérica.

\begin{abstract}
(A) MTERRVPFSLLRGPSWDPFRDWYPHSRLFDQAFGLPRLPEEWSQWLGGSS WPGYVRPLPPAAIESPAVAAPAYSRALSRQLSSGVSEIRHTADRWRVSLDVNH FAPDELTVKTKDGVVEITGKHEERQDEHGYISRCFTRKYTLPPGVDPTQVSSS LSPEGTLTVEAPMPKLATQSNEITIPVTFESRAQLGGPEAAKSDETAAK (B) MDIAIHHPWIHRPFFPFHSPSRLFDQFFGEHLLESDLFPTSTSLSPFYLRPPSFL RAPSWFDTGLSEMRLEKDRFSVNLDVKHFSPEELKVKVLGDVIEVHGKHEER QDEHGFISREFHRKYRIPADVDPLTITSSLSSDGVLTVNGPRKQVSGPERTIPIT REEKPAVTAAPKK

(C)

MADGQMPFSCHYPSRLRRDPFRDSPLSSRLLDDGFGMDPFPDDLTASWPDW ALPRLSSAWPGTLRSGMVPRGPTATARFGVPAEGRTPPPFPGEPWKVCVNV HSFKPEELMVKTKDGYVEVSGKHEEKQQEGGIVSKNFTKKIQLPAEVDPVTVF ASLSPEGLLIIEAPQVPPYSTFGESSFNNELPQDSQEVTCT
\end{abstract}

Figura 1. Secuencia primaria de Hsp27 (A), alfa B-cristalina (B) y HspB8 (C). En negrilla se indican los aminoácidos que conforman el dominio alfa cristalino (secuencia altamente conservada en las sHps). 

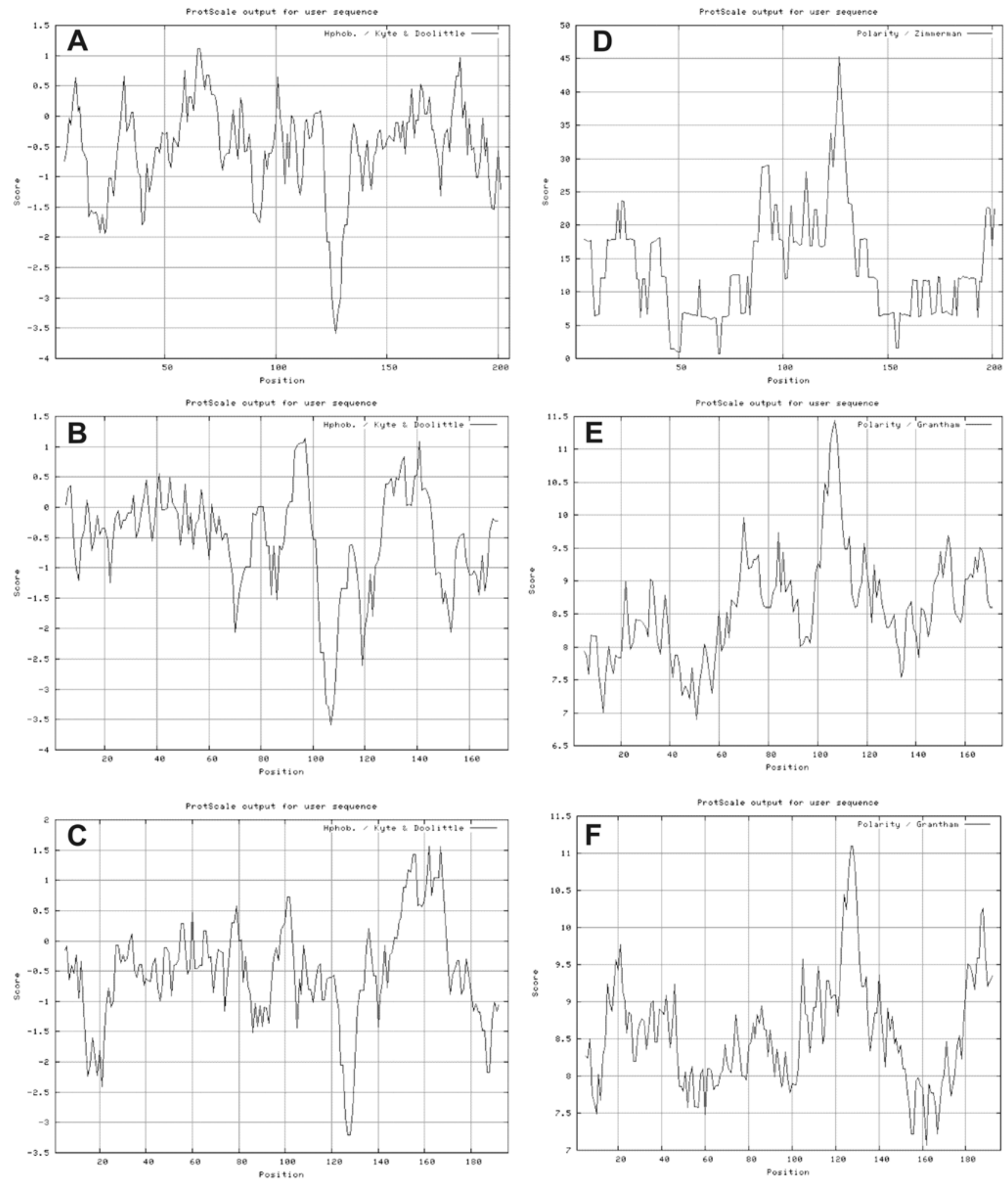

Figura 2. Perfiles de hidrofobicidad y polaridad. Hidrofobicidad según algoritmo de Kyte-Doolitle (escala de -3.5 a 4.2 , en el eje de las ordenadas y posición de los aminoácidos en el eje de las abscisas). A: Hsp27, B: $\alpha$ B-cristalina, C: HspB8. Polaridad según algoritmo de Zimmerman (escala de 0.00 a 52, en el eje de las ordenadas y posición de los aminoácidos en el eje de las abscisas). D: Hsp27, E: $\alpha$ B-cristalina, F: HspB8. 

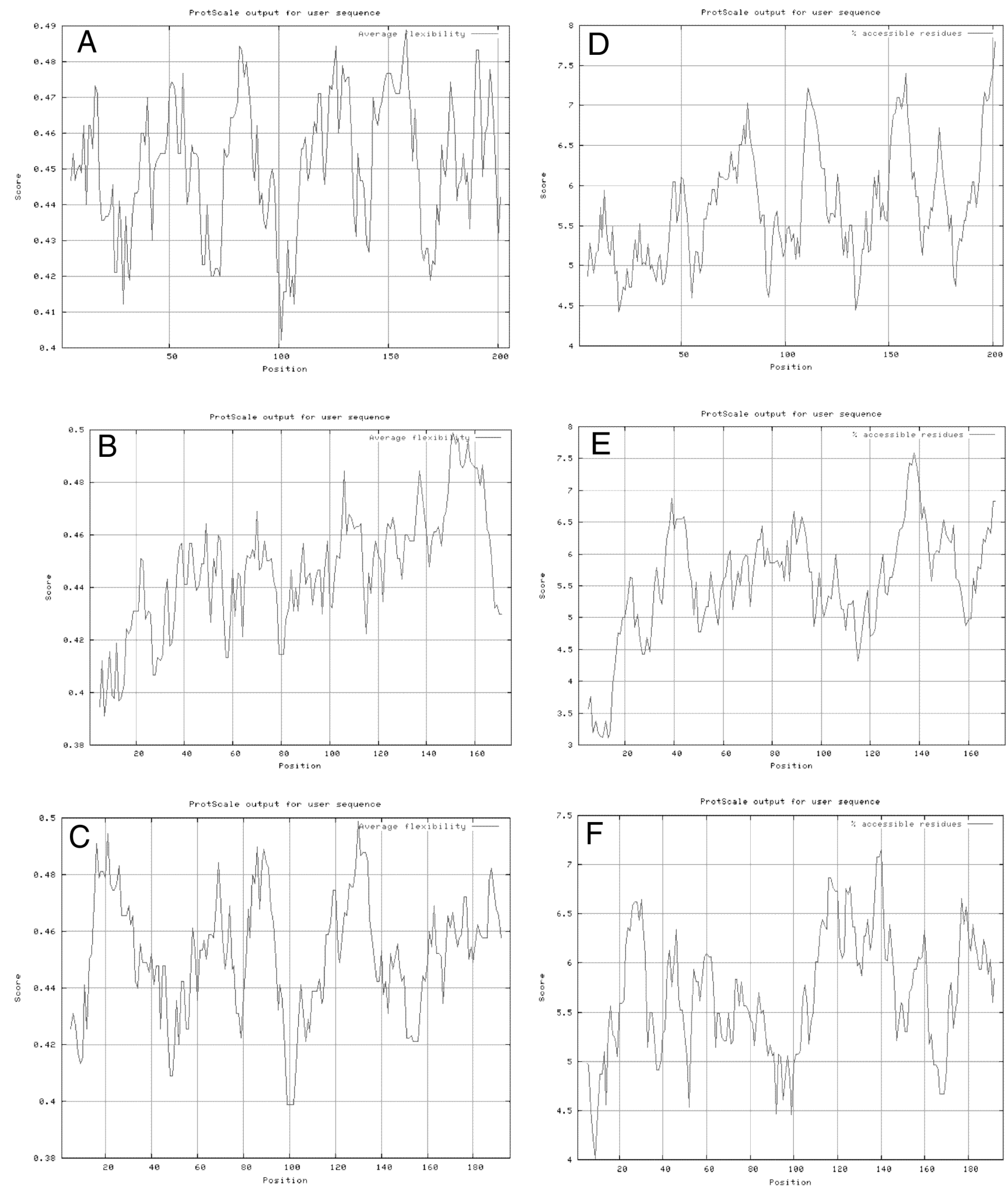

Figura 3. Perfiles de flexibilidad y accesibilidad. Flexibilidad según algoritmo de Bhaskaran (escala: 0.300 a 0.540 en el eje de las ordenadas y posición de los aminoácidos en el eje de las abscisas). A: Hsp27, B: $\alpha B$-cristalina, C: HspB8. \% accesibilidad (escala: 0.300 a 0.900 en el eje de las ordenadas y posición de los aminoácidos en el eje de las abscisas). D: Hsp27, E: $\alpha B-$ cristalina, F: HspB8. 

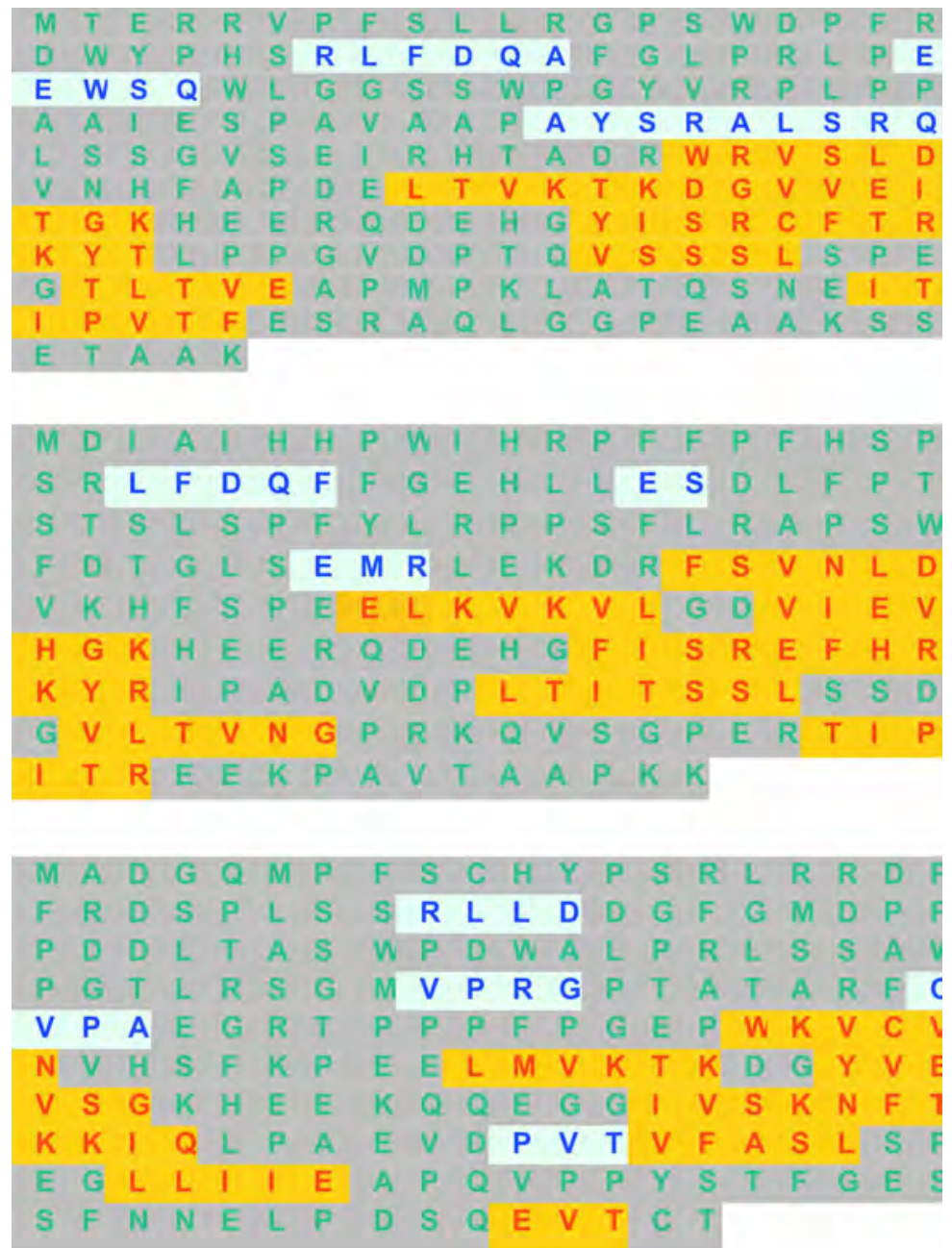

Figura 4. Predicción secundaria de las proteínas humanas Hsp27, alfa B-cristalina y HspB8. La predicción es producto del consenso de los programas GOR 4, nnPred, Sspro, APSSP2, JPredict, Porter, Prof, SOPMA, HNN y Psi-Pred para predicción de estructura secundaria. Helice (azul); Coil (gris); hoja plegada (amarillo). Superior: Hsp27. Centro: alfa B-cristalina. Inferior: HspB8 .

\section{Perfiles de antigenicidad}

Los perfiles de antigenicidad fueron establecidos empleando el programa JaMBW Chapter 3.1.7 el cual utiliza una escala de hidrofilicidad, asignándole un valor a cada uno de los aminoácidos, con variaciones entre -3.4 (Triptofano) y 3.0 (Arginina). El algoritmo promedia valores de hexapeptidos que son luego promediados a lo largo de toda la secuencia y considera que los picos de la curva están asociados a determinantes antigénicos (19). Los perfiles así obtenidos son mostrados en la figura 6 y la ubicación de zonas antigénicas en el modelo de estructura terciaria de cada proteína se muestran en la figura 7.
Hsp27: El perfil de antigenicidad muestra regiones antigénicas de la proteína entre los aminoácidos en posición 120-130 y entre los aminoácidos en posición 190-200.

$\alpha B$-cristalina: El perfil de antigenicidad muestra que regiones antigénicas se ubican entre los aminoácidos en posición 60-70, 100-110 y 158-168.

HspB8: El perfil de antigenicidad muestra que regiones antigénicas se ubican entre los aminoácidos en posición $10-20$ y $120-130$. 

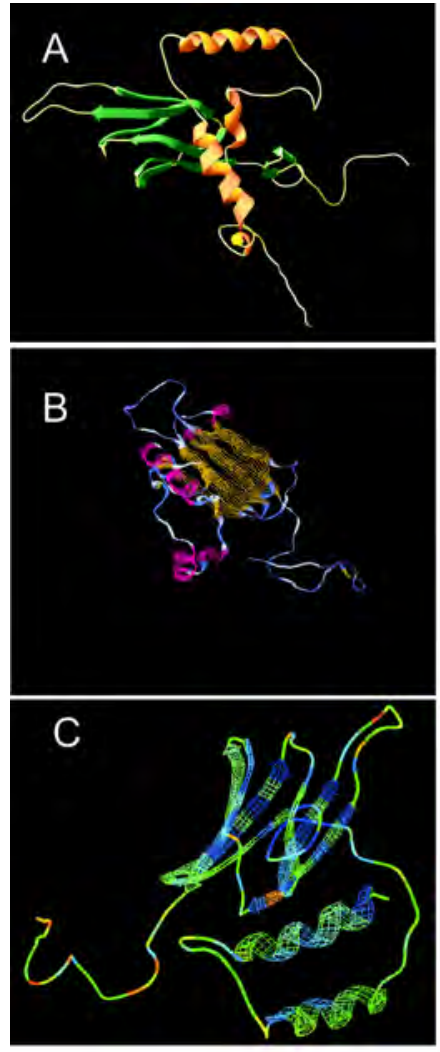
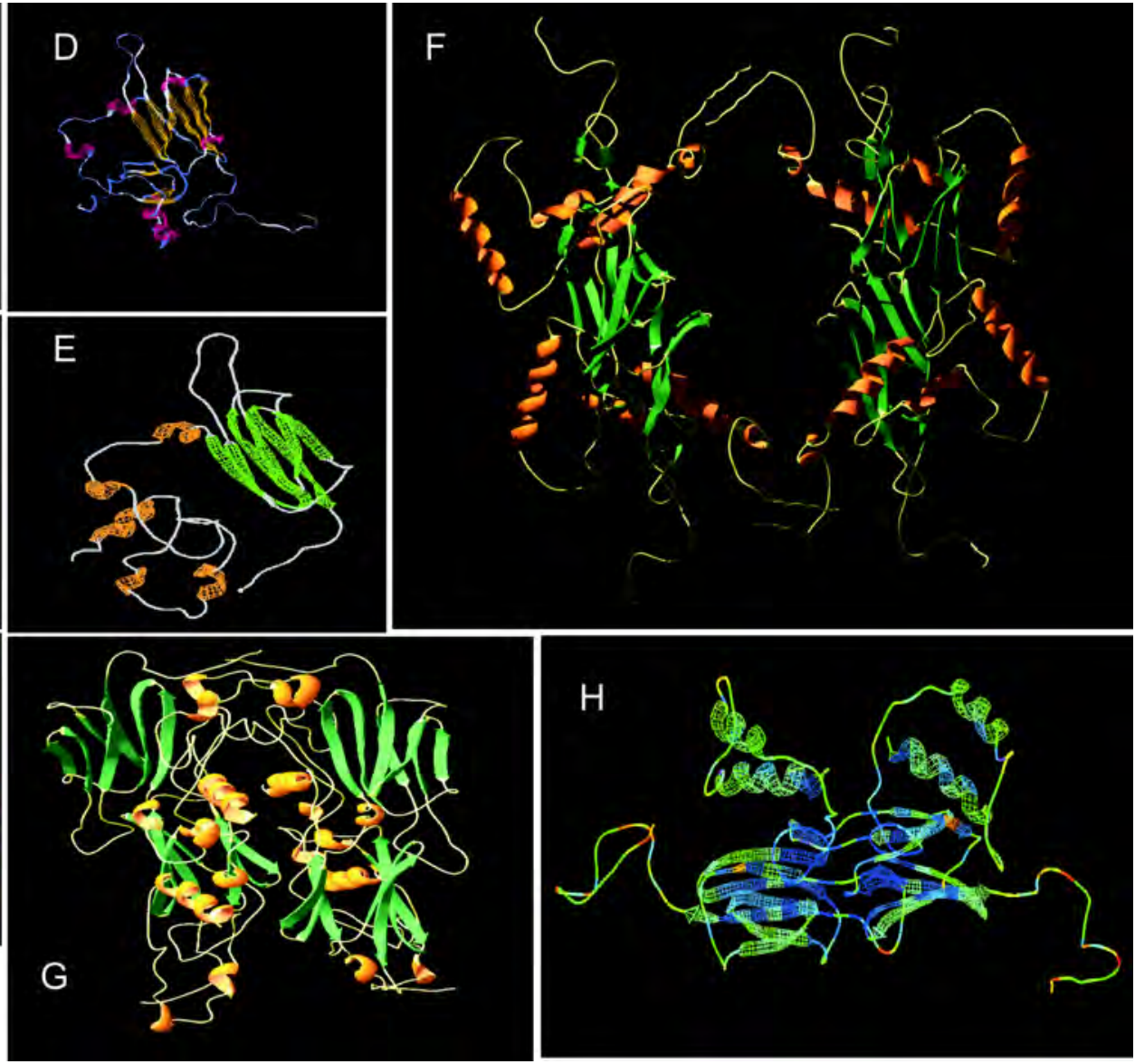

Figura 5. Modelos de estructura terciaria de las proteínas humanas Hsp27, $\alpha B$-cristalina y HspB8. A: Modelo de estructura monomérica de Hsp27. B-C-E: Modelo de estructura terciaria monomérica de $\alpha B$-cristalina. D: Modelo de estructura terciaria monomérica de HspB8. F: Modelo de estructura tetramérica de Hsp27. G: Modelo de estructura tetramérica de $\alpha$ B-cristalina. H: Modelo de estructura dimérica de HspB8 humana. Visualización con RasMol 2.6 (B-D), visualización con Spdbv 4.0.1 (C-E-H), visualización con POV 3.6 (A-F-G),

\section{Análisis de mutaciones}

Los resultados de los perfiles de hidrofobicidad, polaridad, flexibilidad y porcentaje de accesibilidad para cada una de las mutaciones analizadas sugieren que en todos los casos las mutación alteran al menos uno de los perfiles estudiados (Tabla 1).

\section{Discusión}

Aunque inicialmente, el choque térmico a nivel celular fue asociado a causas ambientales, el mismo efecto es provocado por situaciones fisiopatológicas y agentes bioquímicos, como la respuesta celular a la infección causada por agentes virales y bacterianos o la exposición a metales pesados, análogos de aminoácidos, pequeñas moléculas farmacológicamente activas y oxidantes. Común a estos tipos de estrés son los efectos asociados al plegamiento, translocación, ensamblaje y degradación de las proteínas. Como consecuencia se produce un incremento de proteínas no plegadas, así como un incremento del desplegaje y formación de agregados de proteínas, lo que induce una respuesta celular mediada por una síntesis elevada de chaperonas moleculares y proteasas, cuyo objetivo es reparar el daño proteico (34). La función principal de las proteínas de estrés es su actividad como chaperonas moleculares. En este sentido, está bien establecido que muchas proteínas no pueden adquirir su conformación nativa sin la asistencia de las chaperonas moleculares (35). Además, las chaperonas moleculares interactúan transitoriamente con 


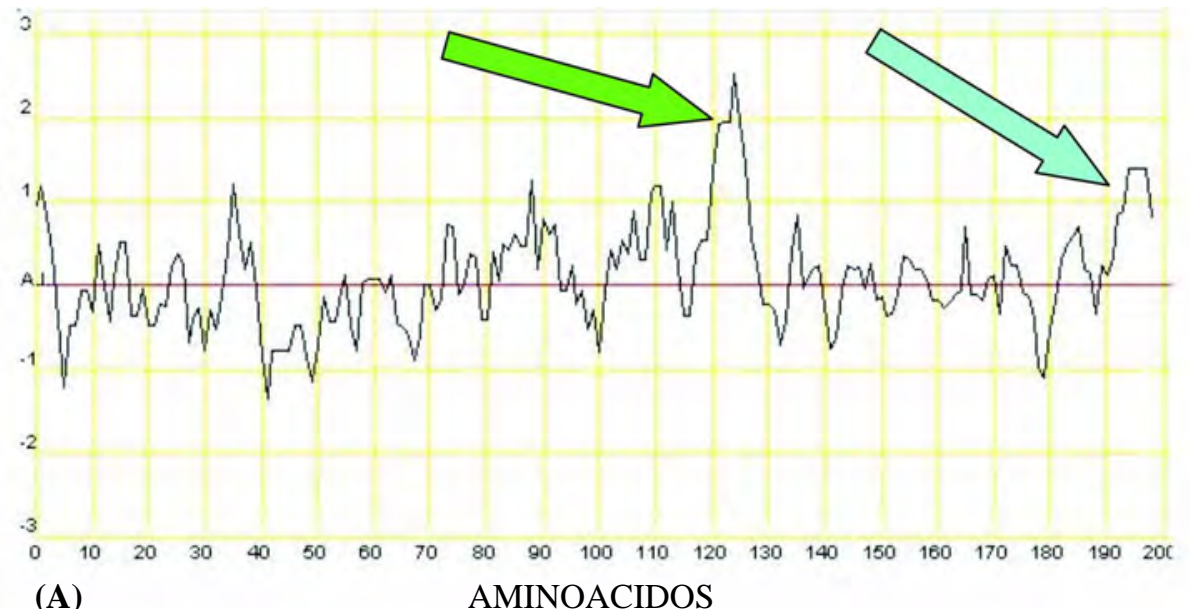

(A)

AMINOACIDOS

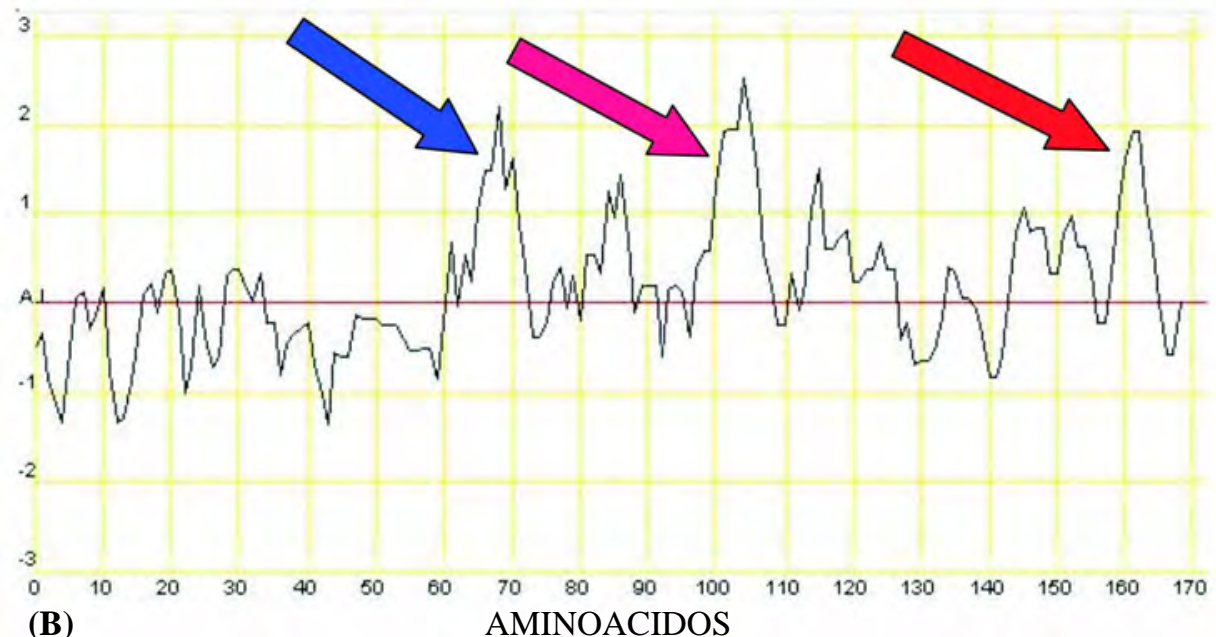

(B)

AMINOACIDOS



Figura 6. Perfiles de antigenicidad de las proteínas humanas Hsp27, $\alpha$ B-cristalina y HspB8. Los perfiles fueron establecidos empleando el programa JaMBW Chapter 3.1.7 (escala de hidrofilicidad: -3.4 a 3.0 en el eje de las ordenadas y posición de los aminoácidos en el eje de las abscisas). (A): Hsp27: se señalan regiones antigénicas comprendidas entre los aminoácidos en posición 120-130 (verde), 190-200 (turquesa). (B): $\alpha$ B-cristalina: se señalan regiones antigénicas comprendidas entre los aminoácidos en posición 60-70 (azul), 100-110 (fucsia) y 158-168 (rojo). (C): HspB8: se señalan regiones antigénicas comprendidas entre los aminoácidos en posición 10-20 (rojo) y 120-130 (verde). 


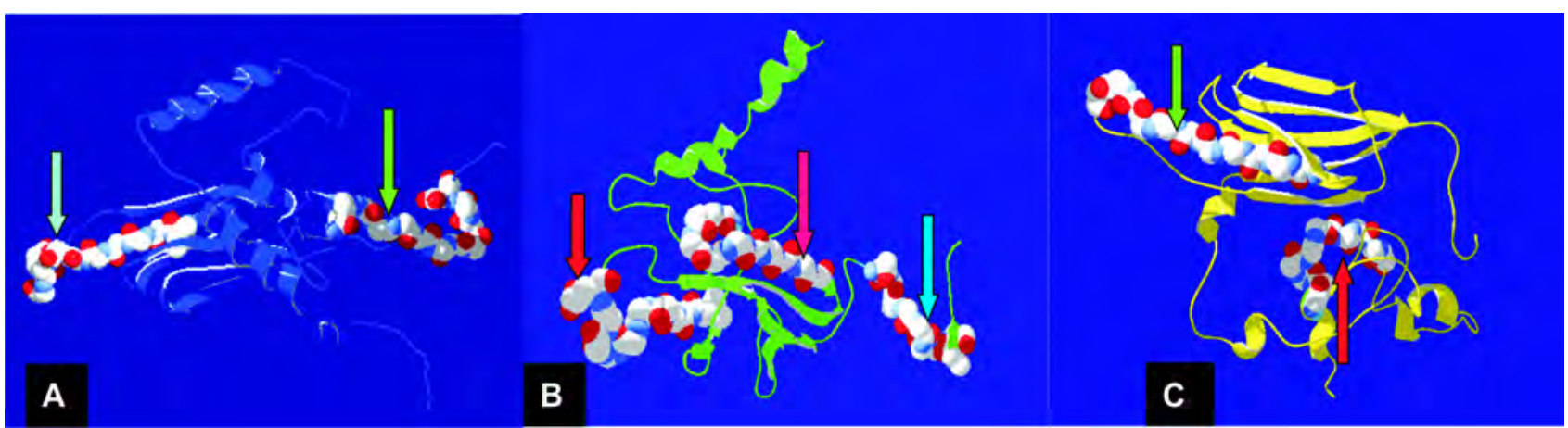

Figura 7. Regiones antigénicas en las proteínas humanas Hsp27, $\alpha B$-cristalina y HspB8 mostradas en los modelos de estructura terciaria. A: Hsp27: aminoácidos 120-130; aminoácidos 190-200. B: $\alpha$ B-cristalina: aminoácidos 60-70; aminoácidos 100-110; aminoácidos 158-168. C: HspB8: aminoácidos 10-20; aminoácidos 120-130. Visualización con el programa POv 3.6.

Tabla 1. Resumen del análisis de los perfiles de hidrofobicidad, polaridad, flexibilidad y \% de accesibilidad de las mutaciones de Hsp27, $\alpha$ B-cristalina y HspB8 humanas.

\begin{tabular}{lllll}
\hline Mutación & Hidrofobicidad & Polaridad & Flexibilidad & Accesibilidad \\
\hline Hsp27 G84R & Disminuida & Sin variación & Sin variación & Sin variación \\
Hsp27 L99M & Sin variación & Sin variación & Sin variación & Disminuida \\
Hsp27 P39L & Sin variación & Sin variación & Disminuida & Sin variación \\
Hsp27 P182L & Aumentada & Sin variación & Disminuida & Sin variación \\
Hsp27 R127W & Sin variación & Disminuida & Disminuida & Disminuida \\
Hsp27 R136W & Aumentada & Disminuida & Disminuida & Disminuida \\
Hsp27 R140G & Aumentada & Disminuida & Sin variación & Sin variación \\
Hsp27 S135F & Sin variación & Sin variación & Disminuida & Disminuida \\
Hsp27 T151I & Aumentada & Sin variación & Sin variación & Disminuida \\
$\alpha B-R 120 G$ & Aumentada & Disminuida & Sin variación & Aumentada \\
$\alpha B-D 140 N$ & Sin variación & Disminuida & Disminuida & Sin variación \\
HspB8 K141N & Sin variación & Sin variación & Sin variación & Disminuida \\
HspB8 K141E & Sin variación & Sin variación & Sin variación & Disminuida \\
\hline
\end{tabular}

otras proteínas a fin de impedir posibles interacciones no apropiadas conducentes a la agregación proteica, estabilizan proteínas nacientes guardándolas en una conformación no plegada con la finalidad de permitir su translocación a través de las membranas y participan en la recuperación de proteínas desnaturalizadas, para lo cual reconocen y se unen selectivamente a proteínas que han perdido su conformación nativa formando complejos relativamente estables (36)
La capacidad de las Hsp de proteger contra la desnaturalización de las proteínas inducida por el estrés térmico y la asociación frecuente de estas moléculas con los agregados proteicos sugieren que están implicadas en el desarrollo de enfermedades degenerativas (15). Además, está bien demostrado que mutaciones en chaperonas moleculares están asociadas a algunas enfermedades (13). El estudio de estas mutaciones constituye el punto de partida para entender cuáles 
son las implicaciones de la ausencia o de las modificaciones de esas chaperonas mutadas. Los hallazgos de diferencias de estructura-función entre las proteínas normales y las mutadas constituyen avances importantes en la búsqueda de develar cómo el producto de la mutación pudiera ser compensado. Sin embargo, en la literatura científica no existen reportes acerca de las estructuras secundaria, terciaria y cuaternaria de las proteínas alfa-B cristalina, Hsp27 y HspB8. Ante la ausencia de conocimientos obtenidos experimentalmente, sobre la conformación nativa de estas moléculas, la elaboración de modelos computacionales para las proteínas normales y mutadas ofrece la posibilidad de tener buenas aproximaciones a las estructuras tridimensionales $(37,38,39)$. A partir de estos modelos se puede obtener información por predicción computacional de algunas de sus propiedades químicas e inferir implicaciones en su comportamiento. La comparación de las estructuras normales y mutadas obtenidas de esta forma, permite conocer la influencia de cada mutación sobre la estructura y función de la molécula normalmente expresada y abre la posibilidad de establecer correlaciones genotipofenotipo (37).

Las predicciones de estructura secundaria (Figuras 4) y terciaria (Figura 5) de las proteínas estudiadas muestra que en todos los casos más del $65 \%$ son regiones en coil, y menos del $10 \%$ en hélice, mientras que en hoja plegada hay regiones entre el 20 y $25 \%$. La prevalencia de conformación en coil hace a estas moléculas más dúctiles y se explica por la necesidad funcional de establecer fácilmente interacciones con las proteínas que van a proteger.

Los perfiles de hidrofobicidad (Figuras 2A, 2B, 2C), muestran que estas proteínas tienen pocas regiones hidrofóbicas, lo cual está en concordancia con sus perfiles de polaridad (Figuras 2D, 2E, 2F), que evidencian que son moléculas muy polares, así como también con los perfiles de accesibilidad (Figuras 3D, 3E, 3F), que indican una elevada proporción de zonas de fácil accesibilidad al medio acuoso. Como cabría esperar son moléculas muy flexibles (Figuras 3A, 3B, 3C), dada su condición de chaperonas moleculares, puesto que la flexibilidad les permite adaptarse más fácilmente a las proteínas substrato a las que se unen para protegerlas. De estas proteínas se conoce por análisis de Western Blot que son proteínas solubles (30), lo cual se corresponde con la información computacional obtenida en este trabajo.

Han sido descritas nueve mutaciones para Hsp27 (G84R, L99M, P39L, P182L, R127W, R136W, R140G, S135F, T151I), dos para alfa B-cristalina (R120G y D140N) y dos para HspB8 (K141N y K141E). A excepción de P39L y P182L de Hsp27, todas esas mutaciones se ubican en el dominio alfa cristalino y tienen en común que afectan el músculo esquelético induciendo miopatías. Adicionalmente pueden comprometer otros órganos, como en el caso D140N y su reportada asociación a la formación de cataratas (17). Fue demostrado que este tipo de proteínas mutadas tienen una actividad disminuida en su función de chaperonas moleculares asociada a la formación de agresomas $(30,35)$, lo que implica disminución en la solubilidad.

El análisis de estructura de estas mutaciones mostró que en todos los casos, al menos uno de los perfiles estudiados está alterado (Tabla 1) y por consiguiente En el caso de Hsp27, la disminución del perfil de flexibilidad inducida por las mutaciones P39L, P182L, R127W, R136W y S135F pareciera ser la responsable de la reducción de la función de chaperona molecular. Para la mutación P182L existe aumento del perfil de hidrofobicidad y disminución del perfil de flexibilidad, lo que sugiere que dicha mutación compromete la solubilidad de la proteína y la pérdida de su actividad de chaperona molecular, lo cual es bien marcado por encontrarse la mutación en la cola flexible (porción de la proteína que la mantiene soluble), esto sin duda afecta severamente sus funcionalidad. De otro lado, la disminución de los perfiles de accesibilidad inducida por las mutaciones L99M, R127W, R136W, S135F y T151I sugiere que la reducción del contacto con el medio acuoso de ciertas zonas de la molécula conlleva a la insolubilidad de la proteína. Así mismo, los aumentos de hidrofobicidad en R140G y T151I, así como las disminuciones de polaridad en R140G y de accesibilidad de T151I sugieren que en presencia de ambas mutaciones Hsp27 afecta su solubilidad. En el caso de la mutación G84R se observó solo una leve disminución de la hidrofobicidad en la zona de la mutación, que aunque leve debe comprometer la solubilidad de la proteína producto del cambio drástico de un aminoácido hidrofóbico por uno polar cargado. Finalmente, la mutación $\mathrm{R} 136 \mathrm{~W}$ es la única que produce alteraciones en todas las características analizadas: hidrofobicidad, polaridad, flexibilidad y accesibilidad, lo que sugiere que es una mutación muy severa que debe afectar drásticamente la estructura y función de la proteína, lo cual podría correlacionarse con la severidad en la enfermedad axonal Charcot Marie Tooth tipo II causada por esta mutación de Hsp27.

Con relación a alfa B-cristalina el aumento del perfil de hidrofobicidad y la disminución del perfil de polaridad inducidos por la mutación R120G explican el hallazgo experimental de la marcada tendencia hacia la agregación $(30,36)$. Por otro lado, los resultados sugieren que la disminución de los perfiles de polaridad y flexibilidad causadas por la mutación D140N son 
responsables de la alteración en su función como chaperona molecular.

En el caso de HspB8 la disminución del perfil de accesibilidad inducida por las mutaciones K141N y K141E parece ser responsable de la insolubilidad de la proteína y la reducción en su función de chaperona molecular, reportado por Carra y colaboradores (40).

Los análisis de estructura primaria se realizaron sobre la secuencia completa de la molécula. Si el análisis se realiza exclusivamente sobre la zona de la mutación, es claro que una variación estructural se debe producir debido al cambio de aminoácido y consecuentemente variaciones en el comportamiento de la proteína. Sin embargo, con la metodología empleada, no fueron detectados eventuales cambios que pudieran afectar algunos perfiles que aparecen sin variación. Sin duda, esto sugiere la necesidad de otros análisis más puntuales con herramientas computacionales más sofisticadas. Como las diferencias son mínimas, es necesario para poderlas establecer y correlacionarlas funcionalmente que sean realizados otros tipos de análisis tales como distancia entre los átomos, desaparición de interacciones electrostáticas y establecimiento de otras nuevas, interacciones de los átomos mutados con átomos de la cola flexible, relación espacial con zonas conservadas evolutivamente (dominio alfa cristalino) y otras.

Los perfiles de antigenicidad (Figura 6 y 7) proporcionaron información sobre zonas antigénicas de utilidad en el corto plazo para la síntesis de péptidos útiles en la producción anticuerpos que serán empleados en ensayos de inmunodetección y cuantificación de las proteínas estudiadas.

La obtención de los modelos computacionales de las proteínas será la base para estudios de bioinformática posteriores tendientes a proponer modelos de interacción entre estas proteínas y sus sustratos y su relación con la formación de agregados proteicos presentes en enfermedades degenerativas. La información que pueda generarse contribuirá decididamente con el propósito de buscar medidas terapéuticas que alivien las dolencias de los pacientes que padecen este tipo de patologías, que si bien no tienen una alta incidencia son devastadoras para quien las padece y tienen un alto costo afectivo y socio-económico.

\section{Conclusiones}

Se elaboraron modelos computacionales de las estructuras secundarias y terciarias de las proteínas humanas Hsp27, alfa B-cristalina y HspB8. Fueron analizadas las mutaciones hasta ahora descritas para estas proteínas encontrándose que todas afectan al menos una de las características estudiadas en la estructura primaria: hidrofobicidad, polaridad, flexibilidad y $\%$ accesibilidad. Los resultados sugieren que estas variaciones son responsables de la disminución en la solubilidad, encontrada experimentalmente, en estas proteínas.

\section{Financiación}

El presente trabajo es producto del proyecto 025-ME-2007 subvencionado por el Centro de Desarrollo Científico Humanístico y Tecnológico (CDCHT) de la Universidad Centroccidental Lisandro Alvarado, Barquisimeto, Venezuela.

\section{Conflicto de intereses}

No existe conflicto de intereses.

\section{Referencias}

1. Linquist S. The heat-shock response. Annual Review of Biochemistry 1986; 55: 1151-91.

2. Kiang JG, Tsokos GC. Heat shock protein $70 \mathrm{kDa}$ : Molecular biology, biochemistry, and physiology. Pharmacology \& Therapeutics 1998; 80:183-201.

3. Frydman J. Folding of newly translated proteins in vivo: the role of molecular chaperones. Annual Review of Biochemistry 2001; 70: 603-47.

4. Kampinga HH, Hageman J, Vos MJ, Kubota H, Tanguay RM, Bruford EA, Cheetham ME, Chen B, Hightower LE. Guidelines for the nomenclature of the human heat shock proteins. Cell Stress and Chaperones 2009; 14(1): 105-11.

5. Ehrnsperger M, Gräber S, Gaestel M, Buchner J. Binding of non-native protein to Hsp25 during heat shock creates a reservoir of folding intermediates for reactivation. The EMBO Journal 1997; 16(2):221-229.

6. De Jong W W, Capers G J, Leunissen J A M. Genealogy of the $\alpha$-crystallin- small heat shock protein superfamily. International Journal of Biological Macromolecules 1998; 22(3-4):51-62.

7. Krief S, Faivre JF, Robert P, Le Douarin B, BrumentLarignon N, Lefrère I, Bouzyk MM, Anderson KM, 
Greller LD, Tobin FL, Souchet M, Bril A. Identificación and characterization of cvHsp. A novel human small stress protein selectively expressed in cardiovascular and insulin-sensitive tissues. The Journal of Biological Chemistry 1999; 274(51):36592-600.

8. Sugiyama Y, Suzuki A, Kishikawa M, Akutsu R, Hirose T, Waye MMY, Tsui S KW, Yoshida S, Ohno S. Muscle develops a specific fprm of small heat shock protein complex composed of MKBP/HspB2 and HspB3 during myogenic differentiation. The Journal of Biological Chemistry 200; 275(2):1095-104.

9. Kappé G, Verschure P, Philipsen RLA, Staalduinen A A, Van de Boogart P, Boelens W C, de Jong W W. Characterization of two novel human small heat shock proteins: protein kinase-related HspB8 and testis-specific HspB9. Biochimica and Biophysica Acta 2001; 1520(1):1-6.

10. Fontaine JM, Rest JS, Welsh MJ, Benndorf R. The sperm outer dense fiber protein is the $10^{\text {th }}$ member of the superfamily of mammalian small stress protein. Cell Stress and Chaperones 2003; 8(1):62-69.

11. Muchowski PJ, Wacker JL. Modulation of neurodegeneration by molecular chaperones. Nature Reviews Neuroscience 2005; 6:11-12.

12. Horwitz J. a-crystallin can function as a molecular chaperone. Proceedings of the National Academy of Sciences of the United State of America 1992; 89(21): 10449-10453.

13. Chávez A, Sáenz H. Implicaciones de las proteínas de choque térmico pequeñas (sHsp/HSPB) en el desarrollo de enfermedades degenerativas. Universitas Scientiarum 2009; 14(1):29-40

14. Arrigo AP, Simon S, Gilbert B, Kretz-Remy C, Nivon M, Czella A, Guillet D, Moulin M, Diaz-Latoud C, Vicart P. Hsp27 (HspB1) and $\alpha \mathrm{B}$ - crystallin (HspB5) as therapeutic targets. FEBS Letters 2007; 581: 3665-74.

15. Sherman MY, Goldberg AL. Cellular defenses against unfolded proteins: a cell biologis think about neurodegenerative diseases. Neuron 2001; 29(1):15-32.

16. Shinohara $H$, Inaguyama $Y$, Goto $S$, Inagaki $T$, Kato K. $\alpha \mathrm{B}$-crystallin and HspB 8 are enhanced in the cerebral cortex of patients with Alzheimer disease. Journal of the Neurological Sciences 1993; 119(2): 203-38

17. Chávez-Zobel AT, Lambert H, Thériault JR, Landry J. Structural instability caused by a mutation at a con- served arginine in the $\alpha$-crystallin domain of Chinese hamster heat shock protein 27. Cell Stress and Chaperons. $2005 ; \mathbf{1 0}(2): 157-166$.

18. Gasteiger E, Hoogland C, Gattiker A, Duvaud S, Wilkins M, Appel RD, Bairoch A. Protein identification and analysis tools on the ExPASy Server. In: John M. Walker (ed): The proteomics protocols handbook. Humana Press. 2005; pp 571-607.

19. Hopp TP, Woods KR. Prediction of protein antigenic determinants from amino acid sequences. Proceedings of the National Academy of Sciences of the United State of America. 1981; 78:3824-8.

20. Soding J. Protein homology detection by HMM-HMM comparison. Bioinformatics 2005; 21: 951-60

21. Garnier J, Gibrat JF, Robson B, Doolittle RF. GOR secondary structure prediction method version IV. Methods Enzymology 1996; 266:540-553.

22. Cuff JA, Clamp ME, Siddiqui AS, Finlay M, Barton GJ. Jpred: A consensus secondary structure prediction server. Bioinformatics 1998; 14:892-893.

23. Kneller DG, Cohen FE, Langridge R. Improvements in protein secondary structure prediction by an enhanced neural network. Journal Molecular Biology 1990; 214: $171-182$

24. Jones DT. Protein secondary structure prediction based on position-specific scoring matrices. Journal Molecular Biology 1999; 292:195-202.

25. Pollastri G, Przybylski D, Rost B, Baldi P. Improving the prediction of protein secondary structure in three and eight classes using recurrent neural networks and profiles. Proteins 2002; 47:228-235.

26. Ouali M, King RD. Cascaded multiple classifiers for secondary structure prediction. Protein Science 2000; 9:1162-1176.

27. Combet C, Jambon M, Deléage G, Geourjon C. Geno3D: Automatic comparative molecular modelling of protein. Bioinformatics 2002; 18:213-214

28. Guez N, Peitisch MC. SWISS-MODEL and the SwissPdbViewer: An environment for comparative protein modeling. Electrophoresis 1997; 18:2714-2723

29. Sayle RA, Milner-White EJ. RasMol: Biomolecular graphics for all. Trends in Biochemical Sciences 1995; 20:374-376

30. Chávez-Zobel AT, Loranger A, Marceau N, Thériault JR, Lambert H, Landry J. Distinct chaperone mechanism can delay the formation of aggresomes by the 
myopathy-causing R120G $\alpha \mathrm{B}$-crystallin mutant. $\mathrm{Hu}$ man Molecular Genetics 2003; 12(13):1609-20.

31. Haslbeck M. sHsps and their role in the chaperone network. Cell Molecular Life Sciences 2002; 59(10):1649-57.

32. Theriault JR, Lambert H, Chavez-Zobel AT, Charest G, Lavigne P, Landry J. Essential role of the NH2-terminal WD/EPF motif in the phosphorylation-activated protective function of mammalian Hsp27. Journal of Biological Chemistry 2004; 279(22):23463-71.

33. Ito H, Kamei K, Iwamoto I, Inaguma Y, Nohara D, Kato K. Phosphorylation-induced change of the oligomerization state of $\alpha \mathrm{B}$-crystallin. Journal of Biological Chemistry 2001; 276(7): 5346-5352.

34. Álvarez M, Espigares E, Rodríguez A, Bueno C, Espigares M. Proteínas de estrés y enfermedades cardiacas. Higiene y Sanidad Ambiental 2007; 7: 228237

35. Fink AL. Chaperone-mediated protein holding. Physiological Reviews 1999; 79(2):425-449.
36. Ehrnsperger M, Gräber S., Gaestel M, Buchner J. Binding of non-native protein to Hsp25 during heat shock creates a reservoir of folding intermediates for reactivation. The EMBO Journal 1997; 16(2):221-229.

37. Sáenz H, Lareo LR, Poutou RA, Sosa C, Barrera LA. Predicción computacional de la estructura terciaria de la Iduronato 2-Sulfato Sulfatasa humana. Biomédica 2007; 27:7-20.

38. Ni M, Yu B, Huang Y, Tang Z, Lei P, Shen X, Xin W, Zhu $\mathrm{H}$, Shen G. Homology modelling and bivalent singlechain Fv construction of anti-HepG2 single-chain immunoglobulin Fv fragments from a phage display library. Journal Biosciences 2008 33:691-7.

39. Wiltgen M, Tilz GP. Homology modelling: a review about the method on hand of the diabetic antigen GAD 65 structure prediction. Wien Med Wochenschr 2009;159:112-25.

40. Carra S, Sivilotti M, Chávez Zobel A, Lambert H, Landry J. HspB8, a small heat shock mutated in human neuromuscular disorders, has in vivo chaperone activity in cultured cells. Human Molecular Genetics 2005; 14(12): 1659-69. 